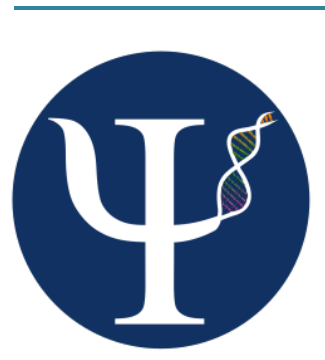

\title{
Imitation and the Developing Social Brain: Infants' Somatotopic EEG Patterns for Acts of Self and Other
} Imitación y desarrollo del cerebro social: Patrones somatotópicos del EEG de niños para sus propios actos y de otros.

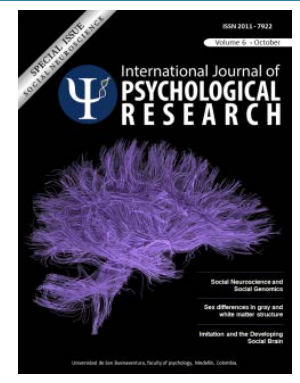

R e s e a r ch

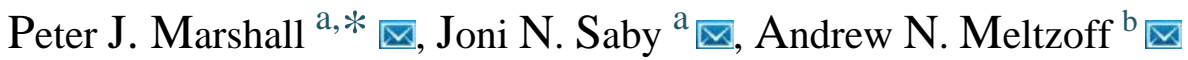

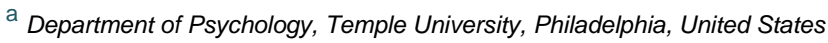 \\ b Institute for Learning \& Brain Sciences, University of Washington, Seattle, United States
}

\section{ARTICLE INFO}

Article history:

Received: 10-09-2010

Revised: 10-10-2013

Accepted: 21-10-2013

\section{ABSTRACT}

A leading question in developmental social-cognitive neuroscience concerns the nature and function of neural links between action perception and production in early human development. Here we document a somatotopic pattern of activity of the sensorimotor EEG mu rhythm in 14month-old infants. EEG was recorded during interactive trials in which infants activated a novel object using their own hands or feet ("execution" trials) and watched an experimenter use her hands or feet to achieve the same goal ("observation" trials). At central electrodes overlying sensorimotor hand areas (C3/C4), mu rhythm power was reduced (indicating greater cortical activation) during infants' execution of hand acts compared to foot acts. For the central electrode overlying the sensorimotor foot area $(\mathrm{Cz})$, mu power was reduced during the execution of foot versus hand acts. Strikingly similar somatotopic patterns were found in both the action execution and observation conditions. We hypothesize that these somatotopic patterns index an intercorporeal mapping of corresponding body parts between self and other. We further propose that infants' ability to identify self-other equivalences at the level of body parts underlies infant imitation and is an ontogenetic building block for the feelings of intersubjectivity we experience when socially engaged with other people.

\section{RESUMEN}

Un asunto importante en neurociencia social-cognitiva del desarrollo tiene relación con la naturaleza y la función de los enlaces neuronales entre la percepción de la acción y la producción de la misma en el desarrollo temprano del ser humano. Aquí documentamos un patrón somatotópico de la actividad de la EEG del Ritmo Mu sensorimotor en bebés de 14 meses de edad. La EEG fue registrada durante ensayos interactivos en los cuales los bebés activaron un objeto nuevo utilizando sus propias manos o pies (ensayos de "ejecución") y vieron a un experimentador usar sus manos o pies para lograr el mismo objetivo (ensayos de "observación"). En los electrodos centrales que cubren las áreas sensoriomotoras correspondientes a la mano (C3/C4), el poder del Ritmo Mu se redujo (indicando una mayor activación cortical) durante la ejecución por los bebés de actos de la mano en comparación con actos del pie. Para los electrodos centrales que cubren el área sensoriomotora correspondiente al pie (Cz), el poder $\mathrm{Mu}$ se redujo durante la ejecución de actos del pie versus actos de la mano. Sorprendetemente, patrones somatotópicos similares fueron encontrados tanto en la ejecución como en la observación de la acción. Tenemos la hipótesis de que estos patrones somatotópicos indican un mapeado intracorpóreo de las partes del cuerpo correspondientes entre uno mismo y los otros. Además, proponemos que la capacidad de los bebés para identificar equivalencias respecto a las partes del cuerpo entre ellos mismos y el otro es la base de la imitación infantil y es un bloque de construcción ontogenético para los sentimientos de intersubjetividad que experimentamos cuando nos comprometemos socialmente con otras personas.

\footnotetext{
* Corresponding author. Peter J. Marshall, Ph.D., Department of Psychology, Temple University, 1701 North $13^{\text {th }}$, Street, Philadelphia, PA 19122, USA, peter.marshall@temple.edu Tel: 215-204-5744.
} 
R E S E A R C H

\section{INTRODUCTION}

Philosophers of mind have argued that intersubjectivity - the feeling of shared understanding and communication experienced by two people-has precursors in shared bodily experiences. The techniques of modern social neuroscience allow this conjecture to be tested in ways that had not been previously available. Two advances enable the relevant tests. The first concerns increased understanding of the brain systems involved in reciprocally mapping action perception and action production. The second derives from developmental psychology, particularly the study of infants.

If we want to know about precursors to adult intersubjectivity, infancy is a good place to start because infancy gives rise to the adult state and because we can examine the nature of the mind and brain before explicit verbal labeling and complex cognitive analysis are operative. Experimental techniques for exploring infant social neuroscience have undergone rapid change in recent years, with continued interest in the utility of electroencephalographic (EEG) methods (e.g., de Haan, 2007). Given that debates about intersubjectivity concern basic aspects of felt communication, sharing, and reciprocity, the ability to record brain activity in preverbal infants during social interaction adds an intriguing level of analysis. One recent focus of infant EEG work has been on the sensorimotor mu rhythm, which occurs in the $6-9 \mathrm{~Hz}$ frequency in the infant EEG at electrode sites overlying sensorimotor cortex (Marshall \& Meltzoff, 2011).

The current study focuses on the neural coding of human goal-directed acts, in particular the overlap between action observation and action execution. This is familiar ground for studies of neural mirroring systems in the animal literature and in adult humans (e.g., Hari \& Kujala, 2009; Rizzolatti \& Sinigaglia, 2010). Scientists have begun to explore the complexities that the consideration of ontogeny brings to such neuroscience studies (Meltzoff, Kuhl, Movellan, \& Sejnowski, 2009). For example, we have used EEG methods to examine changes in the mu rhythm while infants watch and socially engage with an adult (Marshall, Saby, \& Meltzoff, 2013; Marshall, Young, \& Meltzoff, 2011; Saby, Marshall, \& Meltzoff, 2012; Saby, Meltzoff, \& Marshall, 2013). The advance in the current study is the collection of EEG signals from infants while systematically varying the means by which an adult and infant achieve a goal during a live, interactive test paradigm.

More specifically, in the current study we arranged a social turn-taking situation in which infants and adults shared the same goal of pressing a button to activate an interesting effect. The protocol was designed in such a way that the button could be pushed by the infant or the adult using either their hands or feet as the effector. This provided four test conditions pertaining to developmental theory: (a) infant execution of a hand act to achieve the goal, (b) infant execution of a foot act to achieve the goal, (c) infant observation of the adult using her hand to achieve the goal, and (d) infant observation of the adult using her foot to achieve the goal.

Although no previous neuroscience study of infants has compared these four conditions, there are relevant findings from both adult and infant work concerning the patterning of responses across sensorimotor cortex during the observation of actions performed by others. In adults, previous work has revealed a somatotopic patterning such that activation in hand areas is increased when an adult executes or observes hand (versus foot) acts. Conversely, neural activity is of greater magnitude at the foot area when the adult executes or observes foot (versus hand) acts. This somatotopic pattern in adults has been reported using multiple techniques including: (a) functional magnetic resonance imaging (fMRI; Blakemore, Bristow, Bird, Frith, \& Ward, 2005; Buccino et al., 2001; Jastorff, Begliomini, Fabbri-Destro, Rizzolatti, \& Orban, 2010; Wheaton, Thompson, Syngeniotis, Abbott, \& Puce, 2004), (b) transcranial magnetic stimulation (TMS; Senna, Bolognini, \& Maravita, 2013), and (c) EEG (Pfurtscheller, Neuper, Andrew, \& Edlinger, 1997; Pfurtscheller, Neuper, \& Krausz, 2000; Yuan et al., 2010).

Despite the potential importance of somatotopy for illuminating the ontogenesis of interpersonal interaction, developmental aspects have not been well studied. In a recent study we found that the sensorimotor mu rhythm in 14-month-olds shows a somatotopic pattern of activity during infants' observation of hand and foot acts (Saby et al., 2013). Specifically, we reported that infants exhibit a greater reduction in mu rhythm power (indicating an increase in sensorimotor cortex activation) at electrodes overlying sensorimotor hand areas (C3 \& C4) when they observed an adult perform an act using her hand, versus using her foot. Conversely, infants showed a greater reduction in mu power over the sensorimotor foot area $(\mathrm{Cz})$ when they observed the adult using her foot versus using her hand.

This neuroscientific evidence fits with behavioral findings from the infant imitation literature showing that infants maintain a representation of the specific effector used by an adult model to fulfill a goal. For example, Meltzoff (1988) demonstrated that after seeing an adult use his head to press the top surface 
R E S E A R C H

of an object, infants also used their own head to respond imitatively. Somatotopic response patterns at the neural level are also compatible with a range of other findings concerning body-part specificity in infant behavioral imitation (Meltzoff \& Moore, 1997; Meltzoff, Williamson, \& Marshall, 2013). Put another way, we already know that infants can and do imitate with the corresponding body part they see used by an adult, but little is known about the neural correlates of this effect. The ability to assess changes in the sensorimotor mu rhythm in the EEG allows an initial foray into uncovering such interpersonal body mapping at the neural level in human infants.

The current study extends our prior work on somatotopic patterns in the infant EEG in two ways. First, we tested whether the infant EEG exhibits a somatotopic pattern during the execution of the infant's own hand and foot actions. Second and more importantly, we evaluated whether the somatotopic pattern in the infant EEG is similar for both execution and observation. The initial exploration of infant somatotopy (Saby et al., 2013), did not involve an action execution condition and therefore neither of these questions was tested empirically. Both lines of inquiry bear on mechanisms subserving infant imitation and broader issues in social neuroscience related to the nature and function of overlapping neural representations in action production and perception.

\section{METHOD}

\subsection{Participants}

Thirty-five 14-month-old infants participated in the study. Families were recruited from a diverse urban environment using commercially available mailing lists. Families were not invited to participate if their infant was born preterm, if both parents were lefthanded, if the infant had experienced chronic developmental problems, or if the infant was on longterm medication. Informed consent was obtained from the infant's parent prior to participation. The EEG analyses were carried out for 15 infants (mean age $=62.8$ weeks, $S D=1.37,10$ male). The remaining 20 infants were excluded because they became excessively fussy early in the experiment ( $n$ $=2$ ), they did not act on the object during execution trials $(n=1)$, or they had an insufficient number of trials (less than 3 ) that were free of movement and EEG artifact in one or more test conditions ( $n=17)$. This rate of data loss is similar to other studies of the infant mu rhythm involving multiple within-subjects conditions (e.g., Reid, Striano, \& Iacoboni, 2011; Stapel, Hunnius, \& Bekkering, 2012).

\subsection{Apparatus}

The test object was a large dome-shaped button mounted on a base (Sensory Dome, Achievement Products). When the dome is pressed, it activates an effect (a musical sequence with confetti spinning inside the dome) until the pressure is released. This object was chosen because it is likely to be unfamiliar, could be easily activated with hands or feet, and was found to be engaging for infants this age in prior work (Saby et al., 2013).

\subsection{Procedure}

Infants were fitted with an EEG cap and were seated on their caregiver's lap in front of two surfaces, a table $(74 \mathrm{~cm}$ high) and a wooden box (29 $\mathrm{cm}$ high). An experimenter and assistant sat to the left of the infant. The test protocol involved a turn-taking game between the infant and the experimenter, with both of them acting on a shared object (i.e., the Sensory Dome). Infants were first given an opportunity to act on the object using either their hand or their foot, which constituted the execution condition. Infants then observed the experimenter act on the same object using the opposite effector, which constituted the observation condition. When the infant used a foot, the experimenter then used her hand, thus the infant and adult achieved the same endstate/goal (pushing the button to activate the toy) but did so using different effectors (hand vs. foot). This procedure allowed us to generalize beyond the Saby et al. (2013) study which was restricted to infant's observation of the adult's act. It also allowed us to examine whether the somatotopic pattern in the infant EEG during the observation condition was based on the adult's demonstration rather than the infant's immediately prior executed movements (which involved the opposite effector to what they were currently observing).

For a randomly selected half of the infant sample, the protocol began with the assistant placing the sensory dome on the table, near the infant's hands. Infants were given $10 \mathrm{~s}$ to use the object (i.e., to press the dome-shaped button and activate the effect) with their hands. The assistant then picked up the object and placed it on the surface near the feet of the experimenter, who attracted the infant's attention and pressed the button with her right foot, which again activated the effect. This turn-taking sequence was repeated in a block of trials for approximately eight minutes (mean duration $=501 \mathrm{~s}$ ). At this point, a second block of trials was initiated in which the 
assistant first placed the object on the surface near the infant's feet. Infants were given $10 \mathrm{~s}$ to press the button with their foot, and this was followed with the experimenter using her right hand to press the button. This second block of trials continued for another eight minutes (mean duration $=459 \mathrm{~s}$ ). Counterbalancing of the order of the effector used by the infant and adult was achieved, because half of the infants were randomly assigned to press the button with their hands in the first block and their foot in the second block, and half were assigned the reverse order.

In terms of the temporal structure of the protocol, the experimenter's target act (i.e., the contact of her hand or foot with the object) occurred about $5 \mathrm{~s}$ after the offset of the phase in which the infant had been acting on the object. There was then a pause of about $15 \mathrm{~s}$ before the next trial (beginning with the presentation of the object to the infant) was initiated.

\subsection{Linking EEG Data to Behavior}

The experimental session was videotaped, with a vertical interval time code (VITC) placed on the video signal that was aligned with the EEG collection to the precision of one NTSC video frame (33 ms). Laboratory control software from James Long Company was used to simultaneously trigger the onset of the EEG collection and the onset of VITC generation. Videos were coded offline for the time point in which the experimenter's or infant's hand or foot first pressed down on the dome. The videos were also coded for infant motor movements during the infant observation epochs. Observation epochs that contained infant hand or foot movements were excluded from subsequent EEG analyses. The laboratory protocol included calibration stimuli that were visible on the video record and which generated a response in the EEG data stream. The comparison of timing from these stimuli enabled precise adjustment of the sampling rate used in the offline EEG analyses, ensuring consistent temporal alignment between the EEG and video signals.

\subsection{EEG Collection and Processing}

EEG was recorded using a lycra stretch cap (ElectroCap International, Inc.) from the following sites: Fp1, Fp2, F3, F4, Fz, F7, F8, C3, C4, T7, T8, P3, P4, Pz, $\mathrm{P} 7, \mathrm{P} 8, \mathrm{O} 1, \mathrm{O} 2$, and the left and right mastoids. Electrode impedances were accepted if they were below $35 \mathrm{k} \Omega$. The EEG signals were amplified using optically isolated, high input impedance ( $>1 G \Omega$ ) custom bioamplifiers (SA Instrumentation) and were digitized using a 16-bit A/D converter (+/- $5 \mathrm{~V}$ input range). Bioamplifier gain was 4000 and the hardware filter (12 dB/octave rolloff) settings were $.1 \mathrm{~Hz}$ (highpass) and $100 \mathrm{~Hz}$ (low-pass). The EEG signals were collected referenced to the vertex $(\mathrm{Cz})$ with an $\mathrm{AFz}$ ground, and were re-referenced offline to an average mastoids reference prior to further analysis. Data processing and analysis was carried out using a combination of the EEG Analysis System (James Long Company) and the EEGLAB toolbox for MATLAB (Delorme \& Makeig, 2004). Segments which contained excessive artifact due to eye blinks and muscle artifact, or in which the EEG signal at the channels of interest (F3, F4, Fz, C3, C4, Cz, P3, P4, $\mathrm{Pz}$ ) exceeded $\pm 250 \mu \mathrm{V}$ were excluded. For the execution trials, there was an average of 6.4 (SD = 3.7) artifact-free trials per participant for the executehand condition and $6.9(S D=2.4)$ trials for the execute-foot condition. For action observation, the number of artifact-free trials averaged $6.1(S D=2.4)$ for the observe-hand condition and $7.5(S D=2.5)$ for the observe-foot condition.

Spectral power in the mu frequency band was computed by means of the MATLAB pwelch function using a Hamming window (50\% overlap). Based on previous work with infants of this age, the mu frequency band was taken as 6-9 Hz (Marshall, BarHaim, \& Fox, 2002). Our prior work on somatotopy (Saby et al., 2013) expressed spectral power relative to a pre-reaching epoch. However, the structure and timing of the current protocol precluded the isolation of a similar reference epoch; hence here we are reporting absolute rather than relative power values.

Separate analyses were performed for the $500 \mathrm{~ms}$ period preceding initial contact with the object (the "reaching" epoch) and the $500 \mathrm{~ms}$ period following the first video frame in which the infant or experimenter pressed down on the dome, triggering its activation (the "pressing" epoch). As in Saby et al. (2013), power over the left and right hand areas (electrodes C3 and C4) was averaged, in part based on the analysis of infants' behavior when using their hands to activate the object. Specifically, infants used a combination of left, right, and both hands $(15 \%, 59 \%$ and $26 \%$ of trials respectively).

\section{RESULTS}

\subsection{Action Execution: Neural Correlates of Infants Producing the Motor Act}

We tested for differences in mu power at central sites during infants' execution of the motor acts using a $2 x$ 2 repeated measures analysis of variance (ANOVA) on electrode site (hand area, foot area) and test condition (infant execute-hand, infant execute-foot). 
For the reaching epoch, there were no significant main effects or interactions for electrode site or test condition. For the pressing epoch, there were no significant main effects, but there was the predicted significant interaction between electrode site and test condition, $F(1,14)=9.54, p=.008$. As shown in Figure 1, the significant interaction is due to differential spatial patterns of mu rhythm power as a function of test condition. Consistent with the prediction of somatotopy, mu power was lower over hand areas (C3/C4) when infants executed the act using their hands, compared to when they used their feet. The opposite pattern was observed over the foot region $(\mathrm{Cz})$, where mu power was lower when infants used their feet compared to when they used their hands. Supplementary analyses examined the possibility of order effects and the regional specificity of the somatotopic pattern. The (randomly assigned) block order had no significant effect on the somatotopic pattern during action execution. In terms of regional specificity, ANOVAs were performed on 6-9 Hz power over the frontal (electrodes F3/F4, Fz) and parietal (electrodes $\mathrm{P} 3 / \mathrm{P} 4, \mathrm{Pz}$ ) regions during the pressing epoch. No significant interaction between electrode site and test condition was found for either the frontal or the parietal regions, $F(1,14)$ $=.018, p>.50 ; F(1,14)=1.00, p>.50$, respectively, suggesting that the somatotopic pattern was specific to the mu rhythm at central sites.

\section{Figure 1.}

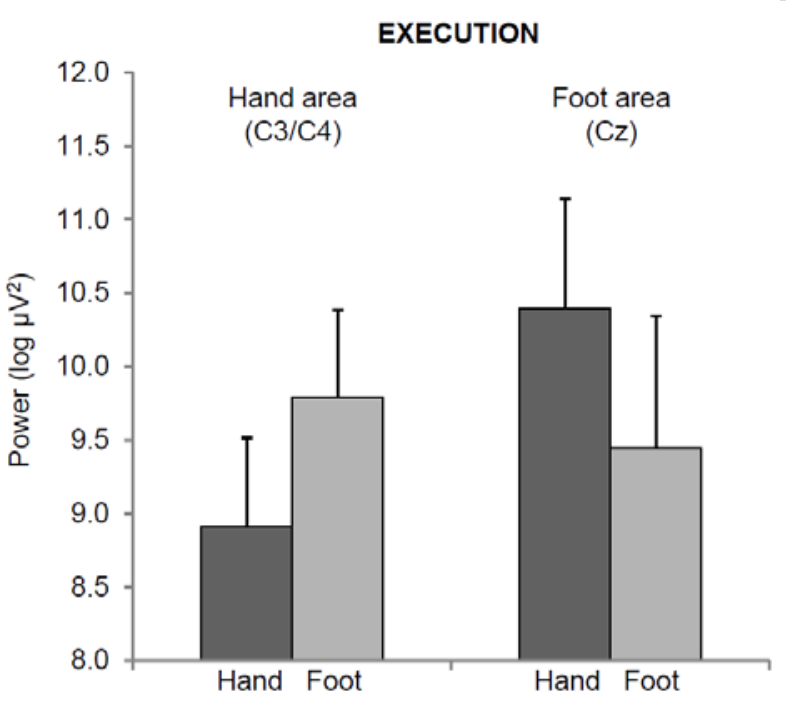

Effector Used by Infant

During Action Execution

Action execution: Mean power in the mu band $(6-9 \mathrm{~Hz})$ at central electrode sites during the infants' execution of hand and foot acts. Compatible with the hypothesis of infant somatotopy, for the electrodes overlying sensorimotor hand areas (C3 \& C4), power was lower for executing hand acts compared to foot acts. For the electrode overlying the foot area $(\mathrm{Cz})$, power was lower during foot acts compared to hand acts. Error bars indicate +1 S.E.M.

\subsection{Action Observation: Neural Correlates of Observing the Adult's Motor Acts}

The same approach was used to analyze the data from the observation period, when the infant was simply watching the experimenter's act. As in the execution period, there were no significant effects in the reaching epoch. For the pressing epoch, there were no significant main effects of electrode site or test condition, but in line with a prediction of somatotopy, there was a significant interaction between these factors, $F(1,14)=6.39, p=.024$. Mu power was lower over infant hand areas (C3/C4) during infants' observation of adult hand acts compared with foot acts. Over the foot area $(\mathrm{Cz}), \mathrm{mu}$ power was lower during infants' observation of adult foot acts than during infants' observation of hand acts (Figure 2).

Again, supplementary analyses examined the possibility of order effects and the regional specificity of the somatotopic pattern. The block order had no significant effect on the somatotopic pattern during action observation. To test for regional specificity, power in the 6-9 Hz band over frontal (F3/F4, Fz) and parietal $(\mathrm{P} 3 / \mathrm{P} 4, \mathrm{Pz})$ regions during the observation of the hand and foot acts was also analyzed. As expected, the interaction between electrode site and test condition was not significant for the frontal, $F(1$, $14)=1.17, p>.05$ or parietal, $F(1,14)=.17, p>$ .05 , regions, again suggesting that the somatotopic pattern was specific to the sensorimotor mu rhythm at central sites.



Action observation: Mean power in the mu band $(6-9 \mathrm{~Hz})$ at central electrode sites during infants' observation of hand and foot acts being carried out by the experimenter. Compatible with somatotopy, power was lower for the electrodes overlying sensorimotor hand areas (C3 \& C4) during the observation of hand acts compared to foot acts. For the electrode overlying the foot area $(\mathrm{Cz})$, power was lower during the observation of foot acts compared to hand acts. Error bars indicate +1 S.E.M. 


\section{DISCUSSION}

The current results show that the mu rhythm in the infant EEG exhibits a somatotopic pattern during the execution of hand and foot actions. Over the sensorimotor hand areas (electrodes C3 \& C4), spectral power in the mu band was lower (reflecting greater activation) during infants' execution of hand actions compared to foot actions, with the opposite pattern being found over the foot area (electrode $C z$ ). The findings from the infant execution condition also provide a "calibration check" on the observation condition. By establishing that the pattern of mu rhythm activity overlying the hand and foot areas shows the predicted changes when infants actually move their hand and foot effectors, this improves one's confidence that the power changes in the mu rhythm are related to activity in the sensorimotor hand and foot areas.

The somatotopic pattern during infants' action execution is consistent with findings from adult studies of the mu rhythm during the production of hand versus foot actions (Pfurtscheller et al., 1997; Pfurtscheller et al., 2000; Yuan et al., 2010). Our findings further demonstrate a striking similarity between the somatotopic pattern in the execution and observation conditions. As noted in the introduction, a variety of studies with adult participants have shown somatotopic patterns of cortical activity during the observation of actions. The present findings add a developmental perspective to the literature on somatotopy by suggesting that a neural mapping of body parts is present during infancy, and as such unlikely to be the product of complex analogical reasoning. We hypothesize that, at least at a primitive level, this intercorporeal mapping provides evidence for a body scheme in young infants-one that links self and other.

The design of the study ensured that the pattern of the EEG response during the observation condition was not wholly attributable to the infant's immediately preceding motor experience. Recall, for example, that when the infant observed the adult using her hand, the immediately preceding trial was one in which infant had used their own foot (and conversely, when the infant observed the adult using her foot, the immediately preceding trial was one in which the infant had used their own hand to activate the object; see "Procedure"). It is also notable that the somatotopic pattern obtained in the current study is consistent with our prior study (Saby et al., 2013), in which infants were not provided any first-hand experience with the object before observing an actor carry out the hand or foot acts, suggesting the robustness of somatotopy in the infant EEG.
In the present study, we performed separate analyses on the reaching and pressing epochs leading up to and following the initial contact with the object. The somatotopic pattern of the mu rhythm was apparent following contact with the object, and not during the reaching epoch (in either the execution or observation conditions). This finding differs from that of our most recent study, in which a somatotopic response pattern was present during the observation of the actor's reach towards the object (Saby et al., 2013); however, in that study we did not examine differences in mu rhythm desynchronization in the epoch following contact of the actor's hand or foot with the object. This cross-study variation concerning the temporal aspects of the somatotopic pattern are likely attributable to key procedural factors. First, our prior investigation of somatotopy used a betweensubjects design such that infants watched the actor use the same effector throughout the course of the entire series of trials (Saby et al., 2013). This consistency meant that it was highly predictable which effector the experimenter was going to use, whereas in the current study both hand and foot acts were shown to infants, making the context more varied and complex. Second, there were differences in the infant's view of the adult's acts. In our prior work, the infant was seated directly facing the experimenter, whereas in the current study infants were seated next to the experimenter, which may have affected infants' ability to observe earlier stages of the adult's reach.

\subsection{Theoretical Insights about Neural Mirroring: Means and Goals}

The nature and function of shared neural representations for action perception and production are at the center of a debate concerning a potential role for neural mirroring systems in action understanding and social cognition (e.g., Rizzolatti \& Sinigaglia, 2010). One key question in this debate is whether such a system is sensitive to the specific means through which a goal is achieved, or whether similar neural reactivity is evoked regardless of the means or manner in which the act was carried out. Evidence from developmental neuroscience on these issues is very limited, although some computational work has attempted to model the development of goal specificity (Thill, Svensson, \& Ziemke, 2011). The current study therefore adds important developmental data to a broader theoretical debate about neural mirroring.

In all four conditions of the current study, the goal was controlled; in all conditions the button was pressed and the audiovisual effect obtained. What varied was how this was done-by hand or by foot. 
R E S E A R C H

The findings clearly show that the specific body part used to perform the act led to specific patterns of mu rhythm activity during both execution and observation. Strikingly, it mattered little whether the infant did the act themselves or simply observed someone else do so, but it mattered a lot whether the goal/endstate was achieved by the hand or by the foot. This suggests that the means used-the specific body part-is not glossed over in the infant's action representation. We endorse the widely held view that goals, endstates, and effects are important in action perception, understanding, and related neural coding. However, there is a crucial additional point uncovered by the current study: The similar somatotopic pattern for both execution and observation suggests that how an act is carried out is also tagged.

\subsection{Putting the Body into Imitation and Intersubjectivity}

This neural sensitivity to the specific body part used is compatible with existing cognitive models of infant behavioral imitation. According to Meltzoff and Moore's (1997) theory of active intermodal mapping (AIM), imitative acts can be differentiated into at least three subcomponents: the body part used, the movement carried out, and the goal or endstate achieved. Concerning the first aspect, Meltzoff and Moore note that the findings of accurate infant imitation in the behavioral literature cannot be accounted for without infants identifying which body part on their own body corresponds to the body part used by the other person. Meltzoff and Moore (1997) hypothesize that this process-which they labeled organ identification-precedes imitation such that "young infants isolate what part of their body to move before knowing how to move it" (p. 183). The patterns of infant neural somatotopy reported in the current study may be part of this hypothesized process.

More speculatively, developmental theorists have argued that infant imitation is a key component of the development of intersubjectivity (e.g., Bråten, 1998; Bruner, 1975; Trevarthen, 1979). Prior to the onset of spoken language, infants communicate through reciprocal actions and gestural turn-taking. One of the longstanding puzzles of intersubjectivity is how these abilities get off the ground. What supports the initial infant feelings that others are "like me" (Meltzoff, 2007, 2013)? We hypothesize that the intercorporeal mapping for body parts of self and other is an ontogenetic building block for intersubjectivity. My hand and your hand are similar; my foot and your foot are similar. When I see you do something I can imitate it in part because I can identify the corresponding body parts across self and other. Investigating somatotopic neural patterns provides a way of exploring the foundations of intersubjectivity at a neural level. Further studies along these lines will enable neuroscience findings to complement and enrich the developmental theories and data that have emerged based on behavioral studies of infants.

\section{ACKNOWLEDGMENTS}

This work was supported by grants from NIH (HD68734) to PJM and NSF (SMA-0835854) to ANM. The authors are grateful to Christina Comalli, Samantha Durkin, Hayley Haaf, Yana Feldman, Melissa Goodwin, Jamie Matthews, Edna Ollquist, Kate Ridge, and Sarah Sanders for assistance with data collection and coding.

\section{REFERENCES}

Blakemore, S. J., Bristow, D., Bird, G., Frith, C., \& Ward, J. (2005). Somatosensory activations during the observation of touch and a case of vision-touch synaesthesia. Brain, 128, 15711583.

Bråten, S. (1998). Intersubjective communication and emotion in early ontogeny. New York, NY: Cambridge University Press.

Bruner, J. S. (1975). From communication to language- A psychological perspective. Cognition, 3, 255-287.

Buccino, G., Binkofski, F., Fink, G. R., Fadiga, L., Fogassi, L., Gallese, V., ... Freund, H. J. (2001). Action observation activates premotor and parietal areas in a somatotopic manner: an fMRI study. European Journal of Neuroscience, 13, 400-404.

de Haan, M. (Ed.). (2007). Infant EEG and eventrelated potentials. New York, NY: Psychology Press.

Delorme, A., \& Makeig, S. (2004). EEGLAB: An open source toolbox for analysis of single-trial EEG dynamics including independent component analysis. Journal of Neuroscience Methods, 134, 9-21.

Hari, R., \& Kujala, M. V. (2009). Brain basis of human social interaction: From concepts to brain imaging. Physiological Reviews, 89, 453-479.

Jastorff, J., Begliomini, C., Fabbri-Destro, M., Rizzolatti, G., \& Orban, G. A. (2010). Coding observed motor acts: Different organizational principles in the parietal and premotor cortex 
of humans. Journal of Neurophysiology, 104, 128-140.

Marshall, P. J., \& Meltzoff, A. N. (2011). Neural mirroring systems: Exploring the EEG mu rhythm in infancy. Developmental Cognitive Neuroscience, 1, 110-123.

Marshall, P. J., Bar-Haim, Y., \& Fox, N. A. (2002). Development of the EEG from 5 months to 4 years of age. Clinical Neurophysiology, 113, 1199-1208.

Marshall, P. J., Saby, J. N., \& Meltzoff, A. N. (2013). Infant brain responses to object weight: Exploring goal-directed actions and selfexperience. Infancy, 18, 942-960.

Marshall, P. J., Young, T., \& Meltzoff, A. N. (2011). Neural correlates of action observation and execution in 14-month-old infants: An eventrelated EEG desynchronization study. Developmental Science, 14, 474-480.

Meltzoff, A. N. (1988). Infant imitation after a 1-week delay: Long-term memory for novel acts and multiple stimuli. Developmental Psychology, 24, 470-476.

Meltzoff, A. N. (2007). 'Like me': A foundation for social cognition. Developmental Science, 10, 126-134.

Meltzoff, A. N. (2013). Origins of social cognition: Bidirectional self-other mapping and the "Like-Me" hypothesis. In M. Banaji \& S. Gelman (Eds.), Navigating the social world: What infants, children, and other species can teach us (pp. 139-144). New York, NY: Oxford University Press.

Meltzoff, A. N., \& Moore, M. K. (1997). Explaining facial imitation: A theoretical model. Early Development and Parenting, 6, 179-192.

Meltzoff, A. N., Williamson, R. A., \& Marshall, P. J. (2013). Developmental perspectives on action science: Lessons from infant imitation and cognitive neuroscience. In W. Prinz, M. Beisert \& A. Herwig (Eds.), Action science: Foundations of an emerging discipline (pp. 281-306). Cambridge, MA: MIT Press.

Meltzoff, A. N., Kuhl, P. K., Movellan, J., \& Sejnowski, T. J. (2009). Foundations for a new science of learning. Science, 325, 284288.

Pfurtscheller, G., Neuper, C., \& Krausz, G. (2000). Functional dissociation of lower and upper frequency mu rhythms in relation to voluntary limb movement. Clinical Neurophysiology, 111, 1873-1879.
Pfurtscheller, G., Neuper, C., Andrew, C., \& Edlinger, G. (1997). Foot and hand area mu rhythms. International Journal of Psychophysiology, 26, 121-135.

Reid, V. M., Striano, T., \& lacoboni, M. (2011). Neural correlates of dyadic interaction during infancy. Developmental Cognitive Neuroscience, 1, 124-130.

Rizzolatti, G., \& Sinigaglia, C. (2010). The functional role of the parieto-frontal mirror circuit: Interpretations and misinterpretations. Nature Reviews Neuroscience, 11, 264-274.

Saby, J. N., Marshall, P. J., \& Meltzoff, A. N. (2012). Neural correlates of being imitated: An EEG study in preverbal infants. Social Neuroscience, 7, 650-661.

Saby, J. N., Meltzoff, A. N., \& Marshall, P. J. (2013). Infants' somatotopic neural responses to seeing human actions: I've got you under my skin. PLOS One.

Senna, I., Bolognini, N., \& Maravita, A. (2013). Grasping with the foot: Goal and motor expertise in action observation. Human Brain Mapping. doi: 10.1002/hbm.22289

Stapel, J., Hunnius, S., \& Bekkering, H. (2012). Online prediction of others' actions: The contribution of the target object, action context and movement kinematics. Psychological Research, 76, 434-445.

Thill, S., Svensson, H., \& Ziemke, T. (2011). Modeling the development of goal-specificity in mirror neurons. Cognitive Computation, 3 , 525-538.

Trevarthen, C. (1979). Communication and cooperation in early infancy: A description of primary intersubjectivity. In M. Bullowa (Ed.), Before speech: The beginning of interpersonal communication (pp. 321-347). New York, NY: Cambridge University Press.

Wheaton, K. J., Thompson, J. C., Syngeniotis, A., Abbott, D. F., \& Puce, A. (2004). Viewing the motion of human body parts activates different regions of premotor, temporal, and parietal cortex. Neuroimage, 22, 277-288.

Yuan, H., Liu, T., Szarkowski, R., Rios, C., Ashe, J., \& He, B. (2010). Negative covariation between task-related responses in alpha/beta-band activity and BOLD in human sensorimotor cortex: an EEG and fMRI study of motor imagery and movements. Neuroimage, 49, 2596-2606. 